\title{
Expression of ADAMTS-7 and ADAMTS-12 in the Nucleus Pulposus During Degeneration of Rat Caudal Intervetebral Disc
}

\author{
Hao $\mathrm{YU}^{1,2)}$ and Yue $\mathrm{ZHU}^{1) *}$ \\ ${ }^{1)}$ Department of Orthopaedics, The First Hospital of China Medical University, Shenyang City, Liaoning Province, China \\ ${ }^{2)}$ Department of Hand Surgery, Fengtian Hospital Affiliated to Shenyang Medical College, Shenyang City, Liaoning Province, China
}

(Received 16 December 2010/Accepted 9 August 2011/Published online in J-STAGE 23 August 2011)

\begin{abstract}
Earlier reports have shown that ADAMTS-7 and ADAMTS-12 (a disintegrin and metalloproteinase with thrombospondin motifs) can bind to and degrade COMP (cartilage oligomeric matrix protein) in cartilage. However, the expression of ADAMTS-7 AND ADAMSTS-12 in both normal intervertebral discs and degenerative intervertebral discs (IVDs) is still unknown and the changes that occur in the degenerating intervertebral disc remain to be determined. The aim of this study was to explore the expression and role of the ADAMTS in the degenerative process of the intervertebral disc. Rat caudal discs were loaded at $1.3 \mathrm{MPa}$ in vivo with an external compression device. The discs were loaded for one week, and and the nuclei pulposi (NPs) were harvested to investigate cell apoptosis and expression of collagen II and aggrecan for confirmation of disc degeneration. The changes of ADAMTS-7 and ADAMTS-12 and COMP were recorded during one week of loading. A second group of rats receiving no load were used as controls. After one week of loading, the NPs suffered degenerative changes as reflected by decreases in cell viability, collagen II, and aggrecan. Dramatic increases in expression of ADAMTS-7 and ADAMTS-12 and COMP fragment were detected after $18 \mathrm{hr}$ of loading; however, this was accompanied by a sharp decrease of COMP expression. These changes were not observed in the control group. The significant increase in COMP possibly suggests a favorable role of COMP in preventing loading and degeneration of matrix; however, this positive effect might be diminished by ADAMTS-7 and ADAMTS-12, whose augmentation were dramatic during disc degeneration.

KEY WORDS: ADAMTS-7, ADAMTS-12, COMP, degeneration, intervertebral disc.
\end{abstract}

doi: 10.1292/jvms.10-0556; J. Vet. Med. Sci. 74(1): 9-15, 2012

Cartilage oligomeric matrix protein (COMP) is a prominent noncollagenous component of cartilage, accounting for $1 \%$ of the wet tissue weight. It is also localized in the tendon, bone (osteoblasts only) and synovium [5-7, 13]. COMP is a $524-\mathrm{kD}$ pentameric, disulfide-bonded, multidomain glycoprotein composed of subunits that are nearly equal in weight (110 kDeach) [20]. It can catalyze collagen fibril formation and to interact with collagen and aggrecan $[2,11,25]$. The interaction between COMP and extracellular matrix (ECM) proteins such as collagen and aggrecan suggests an important role of COMP in modulating ECM composition.

A disintegrin and metalloproteinase with thrombospondin motifs (ADAMTS) is a recently identified metalloproteinase family that can degrade the ECM. First identified in 1997, the members of the ADAMTS family have significant biological relevance [23]. Among them, ADAMTS-7 and ADAMTS-12 directly associate with and degrade COMP $[1,10,14,19]$. ADAMTS-7 is expressed in the bone, cartilage, synovium, tendon and ligament [15], and ADAMTS12 is expressed in the cartilage, synovium and tendon [14], all of which contain COMP $[8,13]$. However, it is not known whether intervertebral discs (IVDs) express ADAMTS-7 and ADAMTS-12, although they are known to

\footnotetext{
* Correspondence to: Zhu, Y., Department of Orthopaedics, The First Hospital of China Medical University, No. 155 Nanjingbei Street, Heping District, Shenyang 110001, China. e-mail: zhuyuedr@163.com
}

(C)2012 The Japanese Society of Veterinary Science contain COMP. Furthermore, the changes in ADAMTS-7, ADAMTS-12 and COMP during disc degeneration remain to be determined.

Histomorphological changes such as fibrosis of the nucleus pulposus (NP) are the results of cellular loss [34] and ECM degradation [9, 27] in the process of IVD degeneration. ECM degradation is a dynamic process involving the loss of proteoglycans, water and type II collagen in the disc matrix [32]. During degeneration, viable disc cells decrease in number $[17,33]$. We created a disc degeneration model in order to fully elucidate the above properties and to determine if there were changes in ADAMTS-7 and ADAMTS-12 during the process of degeneration. To our knowledge, this is the first study evaluating expression of ADAMTS-7 and ADAMTS-12 in the NP. We investigated the presence of ADAMTS-7, ADAMTS-12 and COMP fragments in the disc and their characteristic variations during the process of disc degeneration.

\section{MATERIALS AND METHODS}

Animals: Sprague-Dawley rats weighing 350-400 g were used for this study. The study was approved by the Animal Research Committee of China Medical University. The rats were randomly assigned to one of 2 groups. In the 1 st group $(n=84)$, the intervertebral disc between the 4th and 5th caudal vertebrae was subjected to a constant compressive stress of 1.3 MPa. Six subgroups with eight rats in each were euthanized with excessive ketamine $(0.2 \mathrm{~g} / 4 \mathrm{ml}$; intraperito- 
neal injection) at 6,12 and $18 \mathrm{hr}$, as well as and 1, 4 and 7 days (d). Rats in the second group ( $\mathrm{n}=24)$ who did not have any compressive stress were subjected to sham surgery and euthanized at the same time points as the 1st group but in smaller subgroups of three each.

Disc degeneration model: Degeneration of a single caudal disc was induced by applying static compression [18]. Rats were anesthetized with ketamine $(4-6 \mathrm{mg} / \mathrm{kg}$; intramuscular). Following palpation to identify the caudal 4-5 disc [3], stainless steel insect pins $(0.4 \mathrm{~mm}$ diameter) were inserted percutaneously through adjacent vertebral bodies using a small drill. Two pins were inserted perpendicularly into each vertebral body. A calibrated elastic loop cut from silicone tubing was then placed around each of the 4 opposing ends of the pins to compress the disc to $1.3 \mathrm{MPa}$ of stress $[17,18,29]$. A plastic cylinder was placed over the loaded disc to prevent the rats from chewing the elastic covering. The rats had free range of movement while the loading device was in place. The discs were loaded for 6,12 and 18 $\mathrm{hr}$ and for 1, 4 and $7 \mathrm{~d}$, and the rats were euthanized using an overdose of ketamine $(0.2 \mathrm{~g} / 4 \mathrm{ml}$; intraperitoneal injection) at the assigned time points. After euthanasia, the nucleus pulposus of the treated disc was harvested immediately for further research.

Apoptosis and cell area density: Apoptotic cells were detected in sections using the terminal deoxynucleotidyl transferase (TdT)-mediated dUTP nick-end labeling (TUNEL) method. The sections evaluated were within 100 $\mathrm{mm}$ of those used for proteoglycan content. A commercial TUNEL kit (Roche Diagnostics, Indianapolis, IN, U.S.A.) was used according to the manufacturer's instructions. Sections were counterstained with Hoechst dye (Sigma, St. Louis, MO, U.S.A.). A microscope was used to visualize the number of apoptotic and total cells in the nucleus, and PCI software (Compix, Cranberry Township, PA, U.S.A.) was used to capture and quantitate images. Counts were performed on one representative section per disc. The cell area density was calculated by dividing the total number of cells by the area of the region in which it was measured.

RNA isolation: NPs from between the 4th and 5th caudal vertebrae were used for RNA extraction. The NPs were carefully separated from the annulus fibrosus. Total RNA was isolated using RNAiso ${ }^{\mathrm{TM}}$ Plus (Takara Bio, Otsu,
Japan) according to the manufacturer's instructions. The amount of RNA was determined using a spectrophotometer (Implen, Munich, Germany).

Reverse transcription polymerase chain reaction ( $R T$ $P C R)$ : RT-PCR was performed using a TaKaRa RNA PCR Kit (AMV) Ver.3.0 (Takara, Otsu, Japan). Five hundred nanograms of total RNA was reverse transcribed and added to a $50-\mu l$ PCR reaction following the manufacturers instructions. The primer sequences are listed in Table 1 . Thirty-five cycles of PCR reactions were performed, with each cycle consisting of three phases: denaturation at $95^{\circ} \mathrm{C}$ for $1 \mathrm{~min}$; annealing for $1 \mathrm{~min}$ at $60^{\circ} \mathrm{C}$ for ADAMTS-7, ADAMTS- 12 and COMP, $56^{\circ} \mathrm{C}$ for collagen II, $61^{\circ} \mathrm{C}$ for aggrecan and $58^{\circ} \mathrm{C}$ for GAPDH; and extension at $72^{\circ} \mathrm{C}$ for 1 min. After amplification, the PCR products were analyzed by electrophoresis on a $2 \%$ agarose gel. They were stained with GeneFinder and viewed on a FluorImager.

Real-time polymerase chain reaction: Total RNA was extracted and isolated as described above. Real-time RTPCR was performed using an ABI Prism 7000 Real-Time PCR System (Applied Biosystems, Foster City, CA, U.S.A.) and a SYBR PrimeScript RT-PCR Two-Step Kit (DDR063S; TaKaRa Bio). Briefly, a 500-ng sample of RNA was mixed with $5 \times$ reaction buffer, oligo $(\mathrm{dT})$-adaptor primers, an RNase inhibitor and an enzyme in accordance with the manufacturer's instructions. The reaction mixture was incubated at $42^{\circ} \mathrm{C}$ for $30 \mathrm{~min}, 99^{\circ} \mathrm{C}$ for $5 \mathrm{~min}$ and $5^{\circ} \mathrm{C}$ for $5 \mathrm{~min}$. The PCR reaction mixture was prepared by mixing $2 \mu l$ of the cDNA with the forward and reverse primers and SYBR Premix Ex Taq II in accordance with the manufacturer's instructions. The PCR cycling conditions were as follows: $95^{\circ} \mathrm{C}$ for $10 \mathrm{sec}, 95^{\circ} \mathrm{C}$ for $5 \mathrm{sec}$ and $60^{\circ} \mathrm{C}$ for $60 \mathrm{sec}$ for 40 cycles. Gene-specific primers for real-time RT-PCR were designed and synthesized by Takara Biotechnology (Dalian, China). A standard melting curve cycle was used to verify the quality of amplification and absence of primer dimer formation. The transcript of GAPDH mRNA was employed as an internal control for RNA quality. For each gene, three independent PCRs from the same reverse transcription sample were performed. The primers used were as follows: 5' CACTGAGGACTGTGAACTGGTTGAA 3' (sense) and 5' ACGCAGCGTCTCACAGAAATTG 3' (antisense) for ADAMTS-7; 5' GTCTGGCAGAAGCTG-

Table 1. Primer sequences used for RT-PCR of mRNA

\begin{tabular}{|c|c|c|}
\hline Gene & Sequence & Product Size \\
\hline Collagen II & 5' GAAGCACATCTGGTTTGGAG 3' (sense) & 428 bps \\
\hline & 5' TTGGGGTTGAGGGTTTTACA 3' (antisense) & \\
\hline Aggrecan & 5' CGCTACGACGCCATCTGCTAC 3' (sense) & $497 \mathrm{bps}$ \\
\hline & 5' GCCTGCTGTGCCTCCTCAAA 3' (antisense) & \\
\hline COMP & $\begin{array}{l}\text { 5' GGGTTACACGGCATTCAA 3' (sense) } \\
\text { 5' CGGGACCTGTAGAGGACTT 3' (antisense) }\end{array}$ & $224 \mathrm{bps}$ \\
\hline ADAMTS-7 & $\begin{array}{l}\text { 5' GCAACGCTATTGATGAGGAAGACC 3' (sense) } \\
\text { 5' TTGGGAAGGGCAGGTGATGTAGGA 3' (antisense) }\end{array}$ & 572 bps \\
\hline ADAMTS-12 & $\begin{array}{l}\text { 5' CGGTTACATTGACATCGGACTT 3' (sense) } \\
\text { 5' GGGTTAGTCACCTGGAATAGTAGC 3' (antisense) }\end{array}$ & $270 \mathrm{bps}$ \\
\hline GAPDH & $\begin{array}{l}\text { 5' GTCTTCACCACCATGGAGAAGGCTG 3' (sense) } \\
\text { 5' TGAGGTCCACCACCCTGTTGCTGTA3' (antisense) }\end{array}$ & 673 bps \\
\hline
\end{tabular}


GTTGAAAG 3' (sense) and 5' ACCAGTGTTTCTGTGACCACCAAG 3' (antisense) for ADAMTS-12; 5'TCACCGACTTCAGGGCCTTC 3' (sense) and 5' CAGGCCAGGGTCACTGTTCA 3' (antisense) for COMP; 5' GAAGTGATGCATGGCATTGAGG 3' (sense) and 5'ATGATGGCGCTGTTCTGAAGG 3' (antisense) for aggrecan; 5' TGGTGGAGCAGCAAGAGCAA 3' and 5' CAGTGGACAGTAGACGGAGGAAA 3' for collagen II; and 5' AGGTCGGTGTGAACGGATTTG 3' and 5' TGTAGACCATGTAGTTGAGGTCA 3' for GAPDH.

Protein extraction and western blotting: NP was harvested and lysed in a RIPA lysis buffer $(50 \mathrm{mM}$ Tris $\mathrm{HCl}$, $150 \mathrm{mM} \mathrm{NaCl}, 1 \% \mathrm{NP}-40,0.5 \%$ sodium deoxycholate, $0.1 \%$ SDS) with a protease inhibitor cocktail containing phenylmethyl sulfonyl fluoride (PMSF) and dithiothreitol (DTT; Sigma) for $60 \mathrm{~min}$. The concentration of the extracted protein was measured using a BCA protein assay (Kaiji, Nanjing, China). Equal amounts $(50 \mu \mathrm{g})$ of tissue protein were electrophoresed at $100 \mathrm{~V}$ for $3 \mathrm{hr}$ in SDS (sodium lauryl sulfate) polyacrylamide gels and transferred to a Hybond-PVDF (polyvinylidene difluoride) membrane (Amersham, Arlington Heights, IL, U.S.A.). The membrane was incubated with the COMP antibody (Abcam, San Francisco, CA, U.S.A.) [30, 31] diluted in phosphate-buffered saline (TBS) containing $0.5 \%$ BSA (bovine serum albumin) and $0.1 \%$ Tween 20 and then the horseradish peroxidase conjugated goat anti-rabbit secondary antibody (Pierce Biotechnology Inc., Rockford, IL, U.S.A.). ECL (enhanced chemiluminescence) reagents were used to detect the signals, according to the manufacturer's instructions (Amersham, Arlington Heights, IL, U.S.A.).

\section{RESULTS}

Loading increased apoptosis: In animals that were subjected to loading, apoptosis occurred within the nucleus pulposus after $1 \mathrm{~d}$ of loading, which was in agreement previously described results [18] (Fig. 1-1). When the loading time was increased to $7 \mathrm{~d}$, extensive cell death was noted in the notochordal cells within the nucleus pulposus (Fig. 12). Few TUNEL-positive cells were observed in the control group (Fig. 1-3).

Disc loading was associated with alterations in ECM protein expressions: The expression trends of RT-PCR and real-time PCR remained similar. The expression of type II collagen showed no apparent differences when the discs were loaded for less than $18 \mathrm{hr}$. After that, the expression gradually decreased. A notable decrease was not observed until after $4 \mathrm{~d}$ of loading (Fig. 2-1). The change in pattern of aggrecan expression after loading was similar to that of type II collagen (Fig. 2-2). COMP gene expression varied with the duration of loading. COMP was upregulated after $18 \mathrm{hr}$ of loading, and its expression then decreased significantly after $4 \mathrm{~d}$ of loading (Fig. 2-3). COMP protein expression, as determined by Western blotting, was consistent with the results of RT-PCR (Fig. 2-4). Moreover, we detected a COMP fragment of approximately $65 \mathrm{kD}$ in size that was suggestive of a degraded form of the COMP monomer. The fragment was enhanced dramatically after the first day of loading (Fig. 2-4).

The temporal regulation of ADAMTS-7 and ADAMTS12 after loading increased significantly after the first day of loading. However, dramatic increases in ADAMTS-12 levels were observed after $7 \mathrm{~d}$ of loading, but no increase in ADAMTS-7 was observed at that time point (Fig. 3-1, 3-2).

\section{DISCUSSION}

In the present investigation, we adopted a rat caudal degenerative disc model and loaded discs to $1.3 \mathrm{MPa}$. This stress level was chosen because it is a representative of disc stress experienced in humans. For example, given the typical disc area of the rat caudal disc $\left(2.8 \mathrm{~mm}^{2}\right)$ and that of the human lumbar spine $\left(1,250 \mathrm{~mm}^{2}\right)[17,18,22]$, the stresses that were applied reflect human spinal loads of lifting moderate weight $(1,500 \mathrm{~N})[17,18]$. The dramatic decrease in type II collagen, aggrecan and cell density after seven days of loading indicated that our degenerative disc model was successful after seven days of loading as previously described [17, 18, 29]. Using our successful animal model, we were the first to examine the expression of ADAMTS7 and ADAMTS-12, and their substrate, COMP, during the process of disc degeneration. Our results indicate that the expression of COMP within the nucleus pulposus is enhanced after $18 \mathrm{hr}$ of loading. Downregulation of COMP subsequently occurs after the $18 \mathrm{hr}$ time point, whereas the peak expressions of ADAMTS-7 and ADAMTS-12 occur at days 1 and 7 of loading, respectively.

Recently, it was shown that ADAMTS-7 and ADAMTS12 can degrade COMP and that they are significantly upregulated in arthritic cartilage compared with normal control samples. Furthermore, in vitro experiments demonstrate that ADAMTS-7 and ADAMTS-12 bind specifically to COMP in articular cartilage explants and have COMPdegrading activity $[14-16,19]$. The studies also indicate that the levels of ADAMTS-7 and ADAMTS-12 are significantly upregulated and COMP fragments are found in the cartilage and synovium obtained from patients with rheumatic arthritis [30]. The same situation is also observed in vascular muscle cells and injured arteries [31]. Considering that the relevance of ADAMTS-7 and ADAMTS-12 and the degeneration of discs induced by compressive loading remain unknown, we wonder about the status of ADAMTS7 and ADAMTS- 12 expression and the role in the disc. The results of our study indicate that ADAMTS-7 and ADAMTS-12 are present in the nucleus pulposus and that their expressions are unchanged in the first $18 \mathrm{hr}$ of loading, concomitant with the dramatic increase in COMP. However, once subjected to longer loading times greater than 18 hr, ADAMTS-7 and ADAMTS-12 were upregulated in the nucleus pulposus and COMP decreased sharply. Furthermore, 65-kD COMP fragments [30, 31] were also detected in the nucleus pulposus concomitant with the increases in ADAMTS-7 and ADAMTS-12. Considering that 


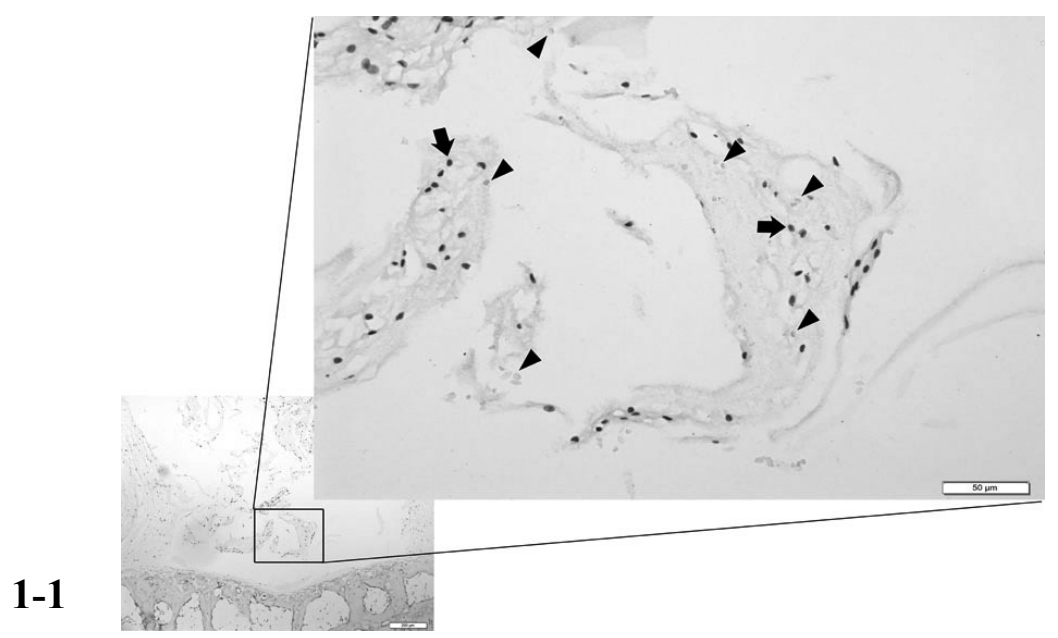

\section{1-2}

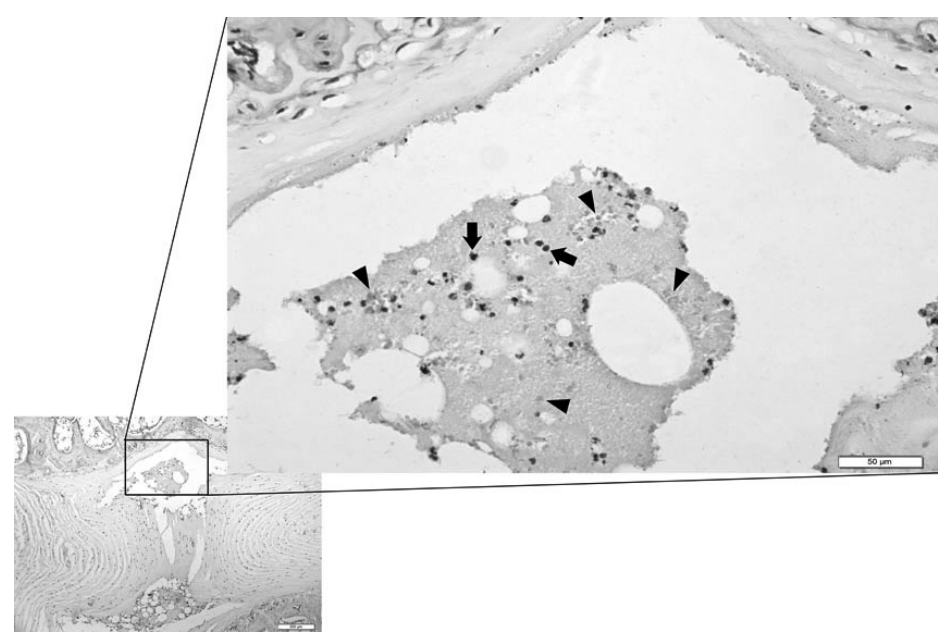

\section{1-3}

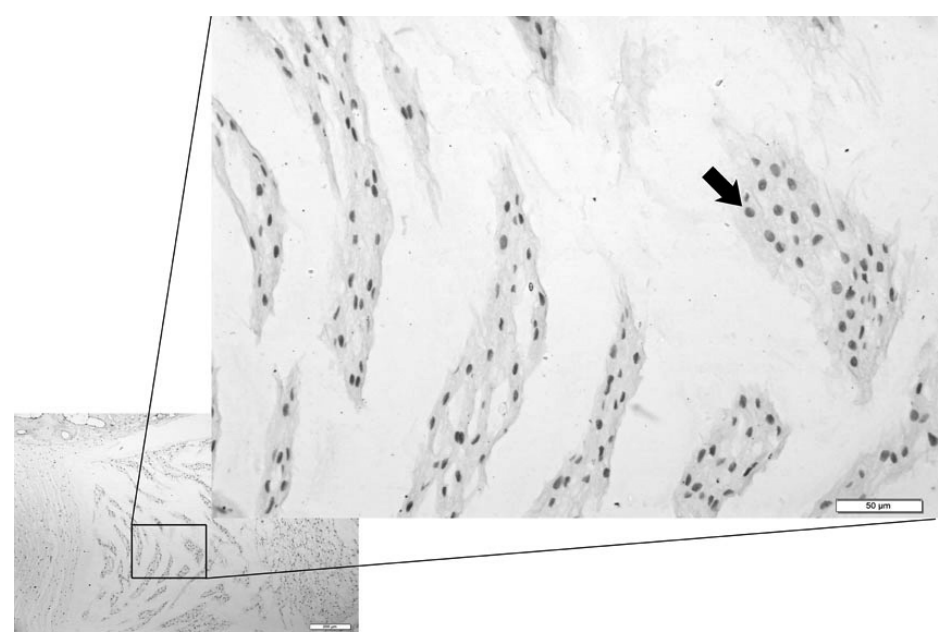

Fig. 1. Nucleus pulposus of a rat tail disc stained with a TdT-mediated dUTP nick-end labeling (TUNEL) assay for apoptosis after $1 \mathrm{~d}$ (Fig. 1-1) and $7 \mathrm{~d}$ (Fig. 1-2) of loading and control (Fig. 1-3). Original magnification: front $\times 400$, rear $\times 200$ (apoptotic cells are marked with arrowheads, and viable cells are marked with arrows). 
2-1
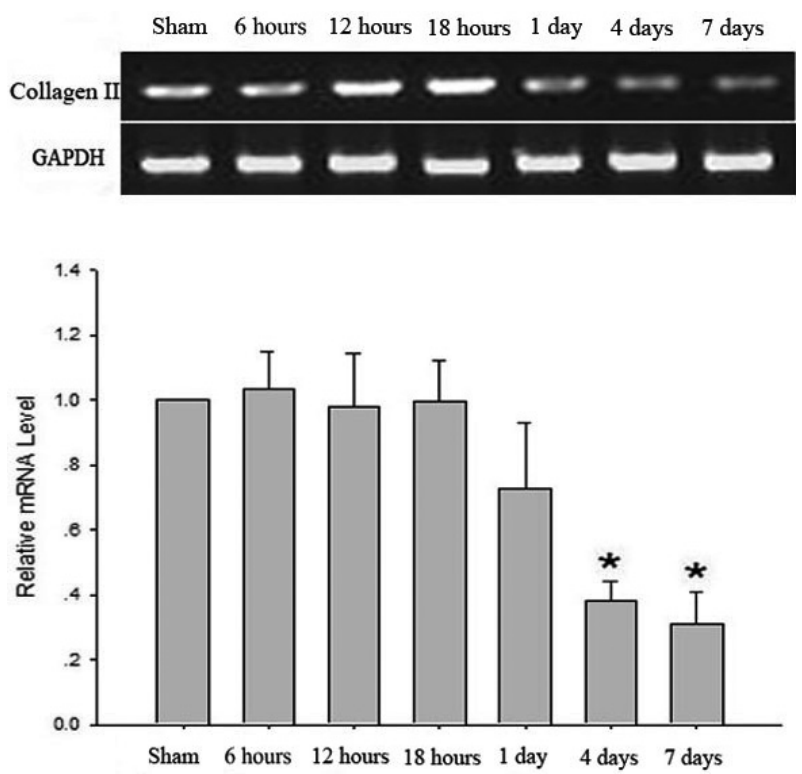

2-3
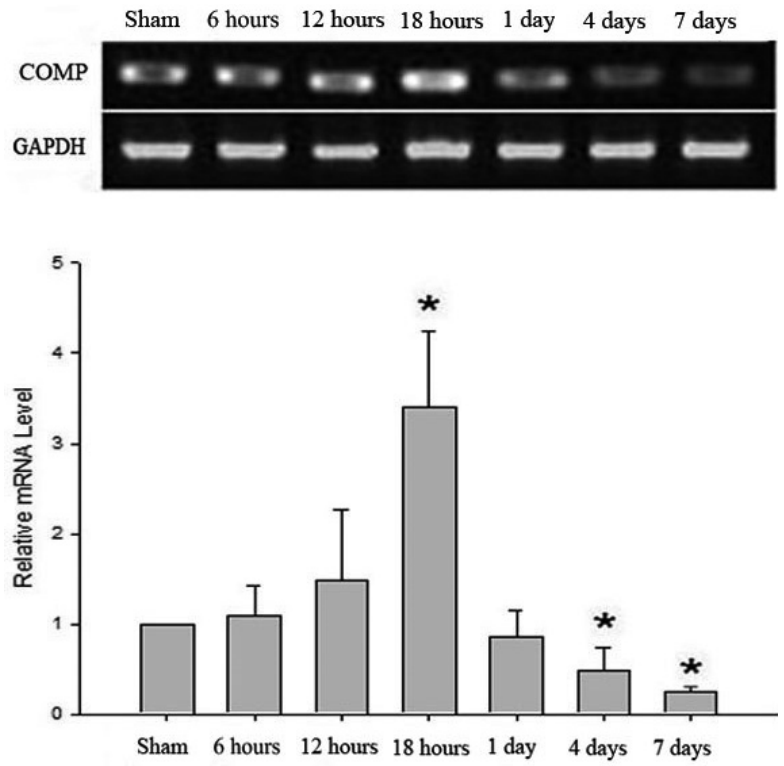

2-2
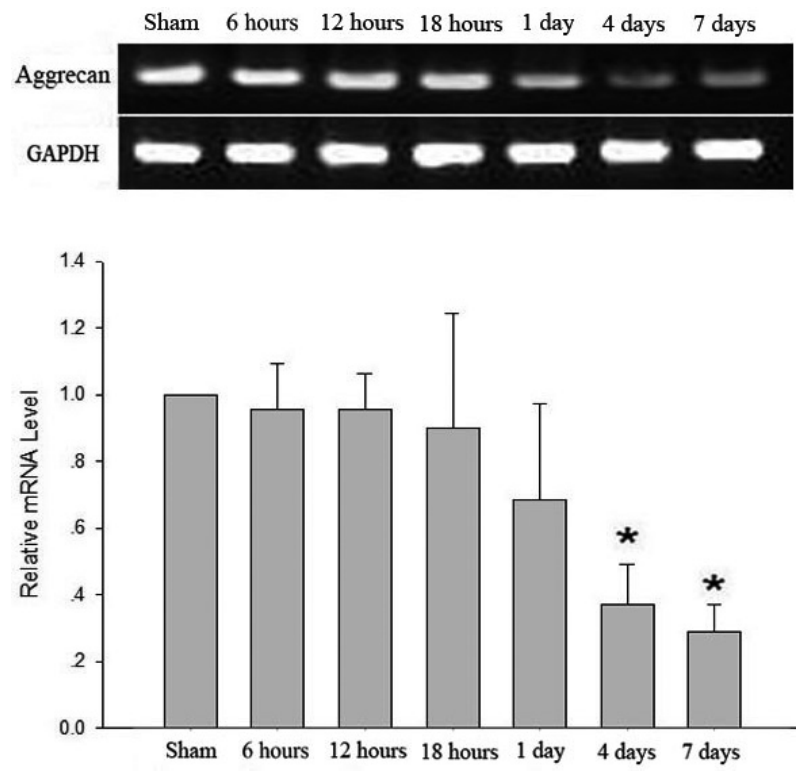

2-4
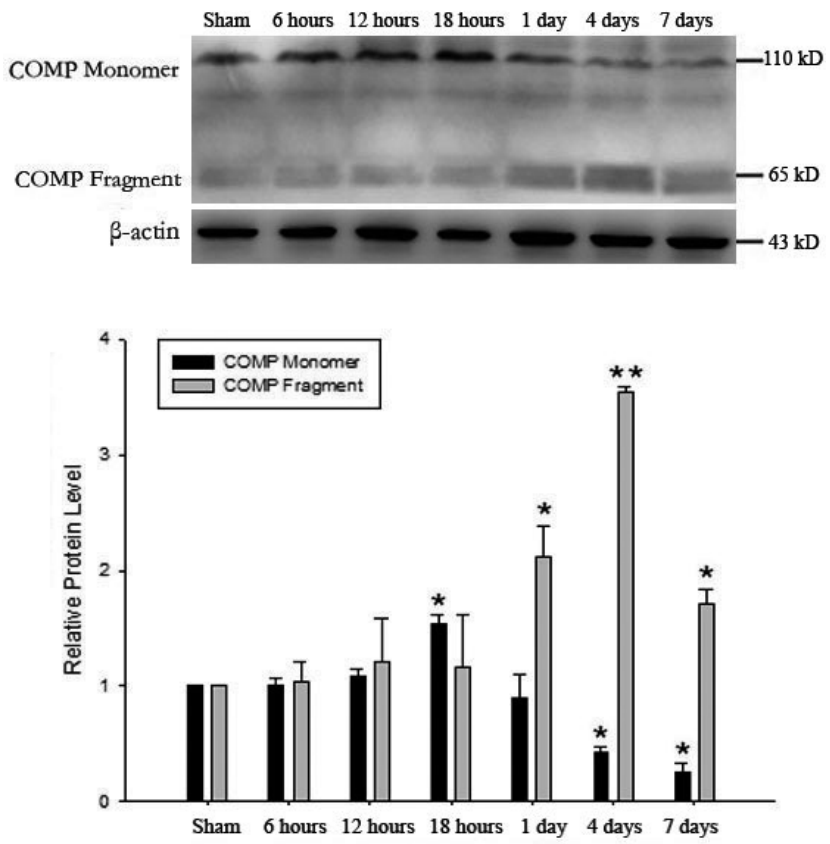

Fig. 2. The variation of major constituents in ECM. The bar charts in Fig. 2-1-2-3 reflect the results of real-time PCR. The expression of type II collagen showed no apparent differences when the loading time was less than $18 \mathrm{hr}$. After that, gradual weakening of expression was observed. A notable decrease was not observed until after $24 \mathrm{hr}$ of loading (Fig. 2-1). The change in pattern of aggrecan expression after loading was similar to that of type II collagen (Fig. 2-2). Upregulation of COMP was observed after $18 \mathrm{hr}$ of loading, after which COMP expression decreased. A statistically significant increase was detected at $18 \mathrm{hr}$ of loading. After that, the intensity of COMP expression was attenuated dramatically (Fig. 2-3). The change in COMP monomer (110 kD) at each time point coincided with the trend of COMP expression in RT-PCR. A 65-kD COMP fragment was detected that showed a statistically significant increase after $1 \mathrm{~d}$ of loading (Fig. $2-4 ; P<0.05$ : vs. control discs. $P<0.01$ : vs. control discs). 
3-1
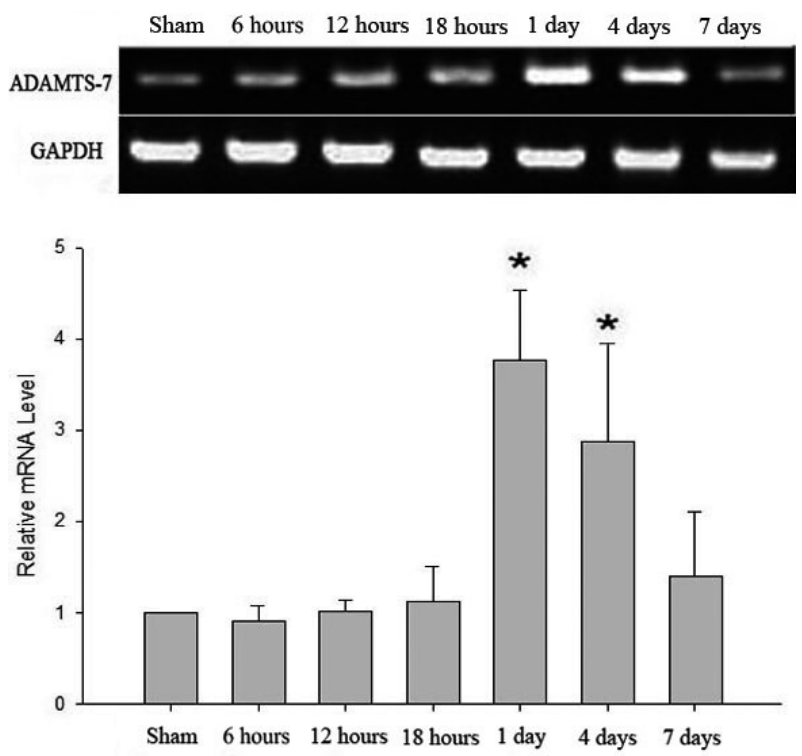

3-2
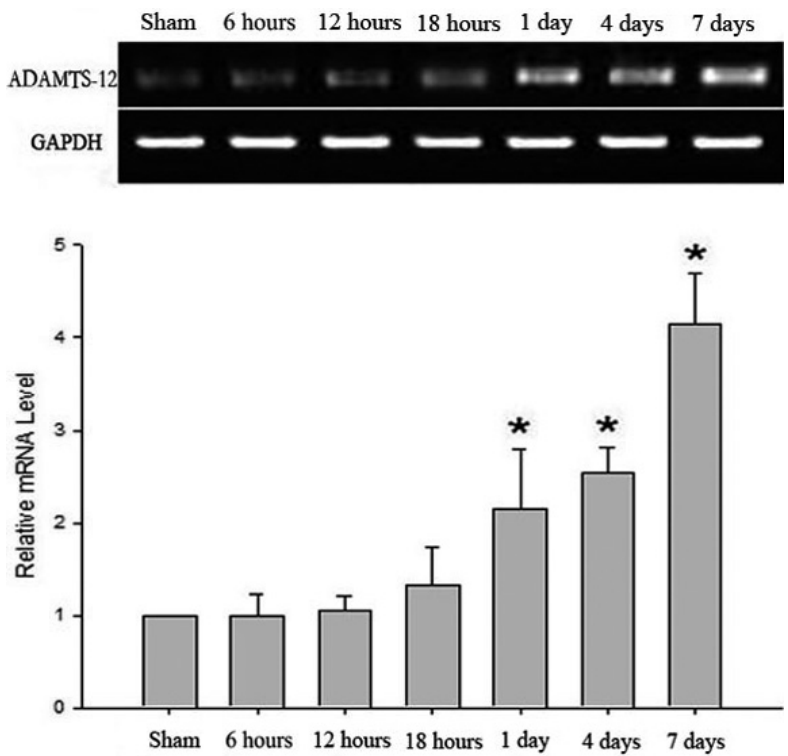

Fig. 3. The mRNA expression of ADAMTS-7 (Fig. 3-1) and ADAMTS-12 (Fig. 3-2) at each loading time and for the control group. The bar chart reflects the results of real-time PCR. ADAMTS-7 and ADAMTS-12 showed a statistically significant increase after loading at $1 \mathrm{~d}$ and $7 \mathrm{~d}$, respectively. The significant increase in the level of ADAMTS-12 lasted for $7 \mathrm{~d}$, which was longer than that of ADAMTS-7 (4 d; $P<0.05$ : vs. control discs).

ADAMTS-7 and ADAMTS-12 can degrade COMP, it is conceivable that higher levels of COMP fragments may be a result of upregulation of ADAMTS-7 and ADAMTS-12 occurring in the degenerating disc. Moreover, we noticed that the load-induced increases in peak expression of ADAMTS-7 (1 d) were earlier than those of ADAMTS-12 ( $7 \mathrm{~d})$. When subjected to $7 \mathrm{~d}$ of loading, the nucleus pulposus still expressed a higher level of ADAMTS-12, but not ADAMTS-7. Accordingly, increases in ADAMTS-7 may occur at earlier stages of disc degeneration, and increases in ADAMTS-12 may occur in the later stages. On the other hand, when the loading duration is excessive and increases beyond the maximum counteracting ability of the matrix, the superfluous portion of loading results in possible metabolism decompensation including changing of osmotic pressure and ion concentration, which eventually evokes cell apoptosis, thus inducing a decrease in ECM proteins including COMP. Consequently, COMP mRNA expression and protein synthesis decreases drastically after $18 \mathrm{hr}$ of loading. The downregulation of COMP caused by cell reduction and degradation by ADAMTS-7 and ADAMTS-12 could be involved in a decrease in nucleus pulposus matrix stability, which could further induce cell reduction by activation of and aggravation of disc degeneration. As a result, ADAMTS-7 and ADAMTS-12 may play a pivotal role in disc degeneration disease progression.

The increase in COMP occurring during loading in our study suggests that COMP is synthesized in vivo in response to load. Some other experiments, albeit performed on cartilage $[12,21,26]$, support this hypothesis. This suggests that the presence of COMP may be necessary to resist load in order for the extracellular matrix to maintain integrity. Matrix integrity and load resistance rely not only on the production of sufficient amounts and structures of proteoglycans and collagen but also on the correct amount and structure of COMP $[4,24,28]$.

This study is the 1 st to report on the expression and kinetic changes in ADAMTS-7, ADAMTS-12 and COMP in the NP during disc degeneration. Our results confirm the presence of ADAMTS-7 and ADAMTS-12 during disc degeneration and indicate that COMP may be degraded by ADAMTS-7 and ADAMTS-12 under conditions where discs are stimulated by consistent loading lasting more than $18 \mathrm{hr}$. The noteworthy increases of ADAMTS-7 and ADAMTS-12 coupled with attenuation of COMP in the nucleus pulposus after loading suggest that ADAMTS-7, ADAMTS-12 and COMP levels are novel markers of disc degeneration.

ACKNOWLEDGMENT. The authors report no conflicts of interest. The authors alone are responsible for the content and writing of the paper.

\section{REFERENCES}

1. Bai, X. H., Wang, D. W., Kong, L., Zhang, Y., Luan, Y., Kobayashi, T., Kronenberg, H. M., Yu, X. P. and Liu, C. J. 2009. ADAMTS-7, a direct target of PTHrP, adversely regulates endochondral bone growth by associating with and inactivating GEP growth factor. Mol. Cell. Biol. 29: 4201-4219.

2. hen, F. H., Herndon, M. E., Patel, N., Hecht, J. T., Tuan, R. S. 
and Lawler, J. 2007. Interaction of cartilage oligomeric matrix protein/thrombospondin 5 with aggrecan. J. Biol. Chem. 282: 24591-24598.

3. Ching, C. T., Chow, D. H., Yao, F. Y. and Holmes, A. D. 2003. The effect of cyclic compression on the mechanical properties of the inter-vertebral disc: an in vivo study in a rat tail model. Clin. Biomech (Bristol, Avon). 18: 182-189.

4. Delot, E., Brodie, S. G., King, L. M., Wilcox, W. R. and Cohn, D. H. 1998. Physiological and pathological secretion of cartilage oligomeric matrix protein by cells in culture. J. Biol. Chem. 273: 26692-26697.

5. Di Cesare, P. E., Fang, C., Leslie, M. P., Tulli, H., Perris, R. and Carlson, C. S. 2000. Expression of cartilage oligomeric matrix protein (COMP) by embryonic and adult osteoblasts. $J$. Orthop. Res. 18: 713-720.

6. Di Cesare, P. E., Fang, C., Leslie, M. P., Della Valle, C. J., Gold, J. M., Tulli, H., Perris, R. and Carlson, C. S. 1999. Localization and expression of cartilage oligomeric matrix protein by human rheumatoid and osteoarthritic synovium and cartilage. J. Orthop. Res. 17: 437-445.

7. Di Cesare, P. E., Carlson, C. S., Stollerman, E. S., Chen, F. S., Leslie, M. and Perris, R. 1997. Expression of cartilage oligomeric matrix protein by human synovium. FEBS Lett. 412: 249-252.

8. DiCesare, P., Hauser, N., Lehman, D., Pasumarti, S. and Paulsson, M. 1994. Cartilage oligomeric matrix protein (COMP) is an abundant component of tendon. FEBS Lett. 354: 237-240.

9. Duance, V. C., Crean, J. K., Sims, T. J., Avery, N., Smith, S., Menage, J., Eisenstein, S. M. and Roberts, S. 1998. Changes in collagen cross-linking in degenerative disc disease and scoliosis. Spine (Phila Pa 1976). 23: 2545-2551.

10. Guo, F., Lai, Y., Tian, Q., Lin, E. A., Kong, L. and Liu, C. 2010. Granulin-epithelin precursor binds directly to ADAMTS-7 and ADAMTS-12 and inhibits their degradation of cartilage oligomeric matrix protein. Arthritis. Rheum. 62: 2023-2036.

11. Halasz, K., Kassner, A., Morgelin, M. and Heinegard, D. 2007. COMP acts as a catalyst in collagen fibrillogenesis. J. Biol. Chem. 282: 31166-31173.

12. Hauser, N., DiCesare, P. E. and Paulsson, M. 1995. The spatial and temporal expression of cartilage matrix protein illustrates the molecular heterogeneity of cartilage. Acta. Orthop. Scand. Suppl. 266: 19-21.

13. Hedbom, E., Antonsson, P., Hjerpe, A., Aeschlimann, D., Paulsson, M., Rosa-Pimentel, E., Sommarin, Y., Wendel, M., Oldberg, A. and Heinegard, D. 1992. Cartilage matrix proteins. An acidic oligomeric protein (COMP) detected only in cartilage. J. Biol. Chem. 267: 6132-6136.

14. Liu, C. J., Kong, W., Xu, K., Luan, Y., Ilalov, K., Sehgal, B., $\mathrm{Yu}$, S., Howell, R. D. and Di Cesare, P. E. 2006. ADAMTS-12 associates with and degrades cartilage oligomeric matrix protein. J. Biol. Chem. 281: 15800-15808.

15. Liu, C. J., Kong, W., Ilalov, K., Yu, S., Xu, K., Prazak, L., Fajardo, M., Sehgal, B. and Di Cesare, P. E. 2006. ADAMTS7: a metalloproteinase that directly binds to and degrades cartilage oligomeric matrix protein. FASEB J. 20: 988-990.

16. Liu, C. J. 2009. The role of ADAMTS-7 and ADAMTS-12 in the pathogenesis of arthritis. Nat. Clin. Pract. Rheumatol. 5: $38-45$.

17. Lotz, J. C., Colliou, O. K., Chin, J. R., Duncan, N. A. and Liebenberg, E. 1998. Compression-induced degeneration of the intervertebral disc: an in vivo mouse model and finite-element study. Spine (Phila Pa 1976). 23: 2493-2506.
18. Lotz, J. C. and Chin, J. R. 2000. Intervertebral disc cell death is dependent on the magnitude and duration of spinal loading. Spine (Phila Pa 1976). 25: 1477-1483.

19. Luan, Y., Kong, L., Howell, D. R., Ilalov, K., Fajardo, M., Bai, X. H., Di Cesare, P. E., Goldring, M. B., Abramson, S. B. and Liu, C. J. 2008. Inhibition of ADAMTS-7 and ADAMTS-12 degradation of cartilage oligomeric matrix protein by alpha-2macroglobulin. Osteoarthritis. Cartilage. 16: 1413-1420.

20. Morgelin, M., Engel, J., Heinegard, D. and Paulsson, M. 1992. Proteoglycans from the swarm rat chondrosarcoma. Structure of the aggregates extracted with associative and dissociative solvents as revealed by electron microscopy. J. Biol. Chem. 267: 14275-14284.

21. Murphy, J. M., Heinegard, R., McIntosh, A., Sterchi, D. and Barry, F. P. 1999. Distribution of cartilage molecules in the developing mouse joint. Matrix. Biol. 18: 487-497.

22. Panjabi, M. M., Goel, V., Oxland, T., Takata, K., Duranceau, J., Krag, M. and Price, M. 1992. Human lumbar vertebrae. Quantitative three-dimensional anatomy. Spine (Phila Pa 1976). 17: 299-306.

23. Porter, S., Clark, I. M., Kevorkian, L. and Edwards, D. R. 2005. The ADAMTS metalloproteinases. Biochem. J. 386: 15-27.

24. Rock, M. J., Holden, P., Horton, W. A. and Cohn, D. H. 2009. Cartilage oligomeric matrix protein promotes cell attachment via two independent mechanisms involving CD47 and alphaVbeta3 integrin. Mol. Cell. Biochem. 338: 215-224.

25. Rosenberg, K., Olsson, H., Morgelin, M. and Heinegard, D. 1998. Cartilage oligomeric matrix protein shows high affinity zinc-dependent interaction with triple helical collagen. J. Biol. Chem. 273: 20397-20403.

26. Smith, R. K., Zunino, L., Webbon, P. M. and Heinegard, D. 1997. The distribution of cartilage oligomeric matrix protein (COMP) in tendon and its variation with tendon site, age and load. Matrix. Biol. 16: 255-271.

27. Sztrolovics, R., Alini, M., Roughley, P. J. and Mort, J. S. 1997. Aggrecan degradation in human intervertebral disc and articular cartilage. Biochem. J. 326 ( Pt 1): 235-241.

28. Vranka, J., Mokashi, A., Keene, D. R., Tufa, S., Corson, G., Sussman, M., Horton, W. A., Maddox, K., Sakai, L. and Bachinger, H. P. 2001. Selective intracellular retention of extracellular matrix proteins and chaperones associated with pseudoachondroplasia. Matrix. Biol. 20: 439-450.

29. Walsh, A. J., Bradford, D. S. and Lotz, J. C. 2004. In vivo growth factor treatment of degenerated intervertebral discs. Spine (Phila Pa 1976). 29: 156-163.

30. Wang, L., Zheng, J., Du, Y., Huang, Y., Li, J., Liu, B., Liu, C. J., Zhu, Y., Gao, Y., Xu, Q., Kong, W. and Wang, X. 2010. Cartilage oligomeric matrix protein maintains the contractile phenotype of vascular smooth muscle cells by interacting with alpha(7)beta(1) integrin. Circ. Res. 106: 514-525.

31. Wang, L., Zheng, J., Bai, X., Liu, B., Liu, C. J., Xu, Q., Zhu, Y., Wang, N., Kong, W. and Wang, X. 2009. ADAMTS-7 mediates vascular smooth muscle cell migration and neointima formation in balloon-injured rat arteries. Circ. Res. 104: 688-698.

32. Yoon, S. T. 2005. Molecular therapy of the intervertebral disc. Spine J. 5: 280S-286S.

33. Zhao, C. Q., Wang, L. M., Jiang, L. S. and Dai, L. Y. 2007. The cell biology of intervertebral disc aging and degeneration. Ageing. Res. Rev. 6: 247-261.

34. Zhao, C. Q., Jiang, L. S. and Dai, L. Y. 2006. Programmed cell death in intervertebral disc degeneration. Apoptosis. 11: 20792088. 